Trans

continentales

\title{
Transcontinentales
}

Sociétés, idéologies, système mondial

\section{La pauvreté urbaine au Japon}

Réalités et représentations

Urban Poverty in Japan : Realities and beliefs

\section{Mélanie Hours}

\section{CpenEdition}

\section{Journals}

Édition électronique

URL : http://journals.openedition.org/transcontinentales/747

DOI : $10.4000 /$ transcontinentales.747

ISBN : 978-2-7351-1563-1

ISSN : 1775-397X

Éditeur

Editions de la maison des sciences de l'homme

\section{Édition imprimée}

Date de publication : 31 décembre 2007

Pagination : 121-138

ISBN : 978-2-200-92397-6

ISSN : $1950-1684$

\section{Référence électronique}

Mélanie Hours, «La pauvreté urbaine au Japon », Transcontinentales [En ligne], 5 | 2007, document 8, mis en ligne le 15 avril 2011, consulté le 08 septembre 2020. URL : http://journals.openedition.org/ transcontinentales/747; DOI : https://doi.org/10.4000/transcontinentales.747 


\title{
La pauvreté urbaine au Japon
}

\author{
Réalités et représentations
}

Mélanie Hours

Le Japon d'après-guerre a su se hisser au rang de deuxième puissance économique mondiale à une vitesse remarquable. Cette spectaculaire croissance a fasciné l'Occident. Le Japon donne aussi l'image d'un peuple homogène, avec un niveau de vie élevé et également réparti dans la population : sa réussite fut caractérisée par l'éradication de la pauvreté, qui n'a pas été remise en cause depuis la crise économique de la fin des années 1980 . Le pays conserve la réputation de ne pas connaître la pauvreté, ce que semble confirmer l'absence d'un taux mesurant ce paramètre. Même dans les statistiques de comparaisons internationales, le cas du Japon reste invariablement non informé. C'est l'un des seuls pays au monde à ne pas posséder un taux de pauvreté : pourquoi quantifier ce qui n'existe pas?

Il a fallu attendre que le Rapport mondial sur le développement humain (élaboré par le Programme des Nations unies pour le développement - PNUD) introduise dans son édition de 2002 un nouvel indicateur de pauvreté humaine ${ }^{1}$ adapté au classement des pays développés, pour avoir un autre point de vue sur la question : le taux de pauvreté concernant le Japon était de 11,2 \% en 2002 et de 11,7 \% en 2005, plaçant le pays en douzième position. À titre de comparaison, la France affiche un

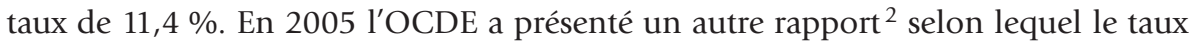
de pauvreté relative au Japon serait «l'un des plus élevés parmi les pays de l'OCDE». Avec un taux de 15,3\%, le Japon se retrouve en cinquième position, derrière le Mexique, les États-Unis, la Turquie et l'Irlande. Cette annonce a été largement relatée dans les médias japonais et elle a suscité la consternation générale, alors que les chiffres du PNUD étaient restés dans l'ombre.

\footnotetext{
1 - L'indicateur IPH-2, calculé à partir des indicateurs de longévité, d'instruction, de conditions de vie et d'exclusion.

2 - OCDE, Shotoku kakusa to hinkon [Disparité de revenus et pauvreté], février 2005.
} 
Évidemment ces taux offrent un véritable contraste avec l'idée d'absence de pauvreté. Après une enquête de terrain effectuée auprès des sans-abri, j'ai été amenée à m'interroger sur les représentations sociales de la pauvreté au Japon. Sans aller jusqu'à parler d'une négation totale de celle-ci, car des mesures d'aide existent, je me suis demandé si, pour un pays qui prétend avoir éliminé la grande misère, l'absence d'un taux de pauvreté national n'était pas plutôt le reflet d'un déni de l'existence de celle-ci. Pour Simmel «c'est l'assistance qu'une personne reçoit publiquement de la collectivité qui détermine son statut de pauvre» ${ }^{3}$. J'ai ainsi étudié plus particulièrement le système d'aide financière aux pauvres, appelé «assistance publique», l'équivalent de notre RMI. Si déni il y a, quelle influence a-t-il sur les mécanismes qui sous-tendent les pratiques d'accès à cette aide? Et plus fondamentalement quel est le statut du droit à l'assistance?

Je présenterai en premier lieu les différentes populations, plus ou moins visibles, touchées par la précarité et la pauvreté. J'aborderai ensuite la question des représentations de la pauvreté à travers la presse et les lois d'assistance, et au sein de la population, pour déceler la présence éventuelle de ce déni et ses conséquences sur la mise en application de l'assistance publique. J'analyserai enfin comment est appréhendé le droit à l'assistance.

\section{Les différents visages de la pauvreté urbaine au Japon}

\section{Les working poor,}

\section{victimes de la précarisation de l'emploi}

Les working poor désignent ceux dont le revenu n'atteint pas le niveau de l'assistance publique. Ces travailleurs pauvres sont en constante augmentation, en partie parce que les entreprises se restructurent en réduisant le nombre de leurs employés permanents. Elles font donc de plus en plus appel aux travailleurs «jetables» : les emplois non réguliers (précaires et à bas salaire) touchent aujourd'hui un tiers des employés. L'accroissement de ce dualisme dans le marché du travail est pour l'OCDE le facteur clé de l'augmentation significative des inégalités ${ }^{4}$.

Au sein de cette nouvelle précarité, la population la plus médiatisée est celle des freeters. Les freeters, selon la définition officielle, sont des personnes entre 15 et 34 ans qui n'ont pas d'emploi à temps plein ou qui sont au chômage de façon intermittente, excepté les femmes au foyer et les étudiants ${ }^{5}$. Le terme est apparu à la fin des années 1980, et vient de la fusion du mot anglais free (libre) et du mot allemand arbeiter (travailleur). Il désignait les jeunes qui refusaient l'emploi permanent pour des postes à temps partiel ou temporaires. On y voyait un changement d'attitude des jeunes face au travail, un rejet du schéma traditionnel de

3 - S. Paugam, "Les trois formes de la pauvreté en Europe», Sciences humaines, n 168, 2006.

4 - OCDE, Economic Survey of Japan 2006, volume 2006, ${ }^{\circ} 13$.

5 - C'est une des définitions possibles : aujourd'hui cette catégorie touche aussi les plus de 34 ans, et plus particulièrement les femmes. 
l'emploi à vie, lequel sous-tend souvent un engagement quasiment inconditionnel envers l'entreprise.

Mais à la suite de la récession économique au début des années 1990, le nombre de postes disponibles pour les futurs diplômés a dramatiquement baissé, remettant en cause le schéma qui leur assurait un poste fixe dès l'obtention de leur diplôme, les entreprises se chargeant de les former à un travail spécialisé. Le nombre de jeunes échouant dans leur transition de l'école au travail a ainsi brusquement augmenté. Les freeters (plus de 4 millions en 2001) ont alors changé d'image; aujourd'hui, même si certains choisissent ce mode de vie, la plupart d'entre eux le subissent et se trouvent face à la précarité qui découle de l'insécurité du travail. Leurs faibles revenus ${ }^{6}$ et le caractère instable et incertain de leurs emplois rendent l'accès au logement difficile. Beaucoup vivent chez leurs parents (ils sont alors qualifiés de "célibataires parasites ${ }^{7}$ »), d'autres dans des hébergements précaires ou encore dans des «manga cafés», ces établissements ouverts vingt-quatre heures sur vingt-quatre où l'on peut lire des mangas et louer à cet effet des petites pièces privées, bien moins chères qu'une chambre dans un petit hôtel.

Depuis fin 2006 les médias se sont centrés sur cette population de «réfugiés des cafés manga/Internet", qui selon une enquête récente concernerait 5400 personnes. Cette focalisation médiatique sur des populations spécifiques (freeters, réfugiés etc.) tend à masquer l'ampleur des symptômes de la précarisation des formes d'emploi. En effet celle-ci touche toutes les catégories de la population, comme les plus de 34 ans (qui n'entrent pas dans la définition officielle des freeters), et en particulier les femmes (50\% des femmes qui travaillent ont un emploi précaire).

Une vision globale de la situation des working poor favoriserait l'identification d'un seul et même phénomène, ce qui permettrait de prendre la mesure de l'extension croissante de la précarité dans la société japonaise actuelle. Cela est d'autant plus indispensable que le plus souvent les emplois non réguliers n'offrent aucune protection sociale (assurance maladie, cotisation pour la retraite), ce qui entraine de graves conséquences pour l'ensemble de ces populations.

\section{Les travailleurs journaliers : entre grande précarité et extrême pauvreté}

Ma rencontre avec les travailleurs journaliers fut le point de départ de mes recherches sur la pauvreté au Japon. J'ai découvert Kamagasaki, le plus grand yoseba (marché du travail journalier ${ }^{8}$ ) du Japon, situé à Osaka : les yoseba sont des univers désolés, comme laissés à l'abandon, et au sein desquels les règles de la société japonaise n'ont plus cours. Pourtant, on y entre en passant par le quartier des affaires, lieu de la vie de tous les jours du citoyen ordinaire. La limite avec ce quartier est si mince que l'on se

6 - Les travailleurs à mi-temps gagnent environ $40 \%$ du salaire horaire d'un travailleur à plein temps.

7 - Parasaito shinguru [célibataires parasites] : expression apparue en 2000 pour désigner les jeunes entre 20 et 35 ans qui vivent chez leurs parents.

8 - Le travail journalier est toujours légal au Japon. 
demande comment ces deux univers peuvent coexister. Kamagasaki est un véritable ghetto au beau milieu de la deuxième ville la plus riche du Japon. On y compte plus de 25000 travailleurs journaliers $^{9}$. Il est estimé qu'entre 100000 et 200000 personnes perçoivent une rémunération journalière au Japon. Il existe d'autres yoseba dans les grandes villes japonaises; Sanya est le plus important à Tokyo.

Si les yoseba diffèrent les uns des autres, des similarités y sont apparentes. Ce sont des espaces liminaires où sont bannies les normes sociales dominantes, des ghettos rarement pénétrés par des personnes étrangères aux lieux. Ce sont aussi des doyagai, des quartiers qui offrent une concentration de logements pas chers (doya), où les hommes payent leur "loyer» à la journée. Les yoseba sont principalement habités par des travailleurs journaliers, des hommes d'âge moyen ou âgés, qui, pour la grande majorité d'entre eux, n'ont plus de liens avec leur famille. Enfin, ce sont des endroits où les populations vivent quasiment en autarcie, frappées d'ostracisme aussi bien par le citoyen "ordinaire» que par les administrations, et tenues à l'écart de la vie civile. Les yoseba offrent cependant un anonymat et une certaine liberté.

Leur rôle est double: il est indissociablement social et économique. Ils constituent avant tout des "trappes pour les paumés, les asociaux ${ }^{10}$ ", ou plus largement, pour tous les laissés-pour-compte de la société japonaise. Les yoseba dévoilent les limites du «miracle économique» japonais, qui trouva ses bases dans une vaste réserve de matériel humain toujours disponible. Les grandes entreprises se reposent sur cette main-d'œuvre «jetable» pour parer à leur vulnérabilité aux fluctuations de l'offre et de la demande. C'est particulièrement le cas de l'industrie de construction, totalement dépendante des travailleurs journaliers, et qui embauche la grande majorité d'entre eux.

Le succès des grandes entreprises de construction du Japon repose beaucoup sur leur habileté à mettre le plus de travailleurs possible à l'écart des contrats de travail. Grâce à un réseau complexe de sous-traitance, les grandes compagnies n'apparaissent jamais en tant qu'employeur direct et ne sont donc pas légalement responsables des diverses infractions au Code du travail (les contrats d'embauche se font à l'oral, sont négociés illégalement et ne donnent droit ni à la sécurité sociale ni au système de retraite).

Le travail journalier est aussi connu sous le nom de "travail des $3 \mathrm{~K}$ », se référant aux termes kitsui pénible, kitanai sale, et kiken dangereux. C'est par définition le travail le plus épuisant physiquement, et le plus dangereux qui soit. Les hommes au-dessus de 50 ans sont rarement embauchés : la priorité est donnée aux plus forts et aux plus jeunes.

En période de récession économique, les travailleurs journaliers perdent toute chance de trouver du travail. Sans logement fixe ni assurance chômage, les liens avec leur famille étant souvent coupés, la fin du travail journalier signifie le plus souvent la vie à la rue. Mais cette fatalité ne dépend pas uniquement des récessions

9 - Voir le travail pionnier d'A. Gonon, Précarité et isolement social, le monde des travailleurs journaliers, Tokyo, Maison franco-japonaise, 1995.

10 - P. Pons, Misère et crime au Japon, Paris, Gallimard, 1999 
économiques. Tout travailleur journalier est en théorie un sans-abri «cyclique» : il suffit de deux jours sans travail pour qu'il ne soit plus en mesure de payer sa nuit dans une doya. C'est cette réalité de la vie du travailleur journalier que sous-entend l'expression abure jigoku, ou «l'enfer du chômage».

Dans les circonstances économiques actuelles, les plus âgés et les moins forts d'entre eux ont échoué plus durablement dans la situation de sans-abri. Aujourd'hui, il y en aurait plus de 2000 dans le yoseba de Sanya, soit $30 \%$ des sans-abri de Tokyo sur une superficie de $1,65 \mathrm{~km}^{2}$; mais c'est sans compter ceux qui, régulièrement, n'ont plus de quoi payer leur doya. Selon une étude de 1999, 46,7 \% des travailleurs journaliers interrogés à Sanya pendant qu'ils cherchaient du travail avaient passé la nuit précédente dehors ${ }^{11}$.

Sanya a toujours eu une connotation négative dans la conscience de la population japonaise. Son destin fut de rassembler les pauvres et tous ceux qui ne trouvaient pas leur place dans la société. Situé au nord-est de Tokyo, Sanya est à quelques stations de Ginza, le quartier des affaires et du luxe à l'occidentale de la capitale. On quitte le monde familier de la ville pour entrer dans un lieu triste où Tokyo semble se terminer. L'impression générale est bien celle du vide: il n'y a pas d'enseignes lumineuses, pas de banques, beaucoup de magasins ont fermé... Le contraste entre l'activité frénétique du reste de la ville et la violente inertie de Sanya est frappant. Les grands axes portent à croire que l'endroit a été déserté. Mais très vite apparaissent des silhouettes, et il faut se glisser dans les petites ruelles pour y trouver des groupes d'hommes assis par terre, en train de boire, de jouer aux cartes, de parier... On découvre alors toute une vie sociale: des petites échoppes, des salles de pachinko ${ }^{12}$, des bars, des magasins spécialisés dans le matériel ouvrier... Sans doute au regard de Tokyo cette vie sociale semble-t-elle "au ralenti», mais elle existe. Sanya est une microsociété, «un autre monde» dans Tokyo.

Les hommes arrivent à Sanya pour toutes sortes de raisons : problèmes de famille, perte d'emploi, faillite, dettes trop lourdes, problèmes avec la justice, maladies mentales... la liste est longue, et propre à chacun. Certains ont abandonné leur famille, d'autres ont été abandonnés. Certains ont trop honte pour rentrer, d'autres se voient raccrocher au nez quand ils appellent leurs proches. Pour ces sans-abri, le processus de rupture de l'inscription dans la société par le travail s'est fait brutalement, sans transition. Commence alors le combat quotidien pour la survie, contre la faim, le froid, les maladies. Le taux de tuberculose à Sanya serait quarante fois supérieur à celui du pays. Certains meurent en silence, de froid par exemple; d'autres périssent de mort violente (meurtres, suicides), parfois sans pouvoir être identifiés.

J'ai effectué une enquête de terrain à Sanya pendant deux ans, et j'ai mené avec les sansabri des entretiens dont le fil directeur était le rapport de ces hommes à leur propre

11 - UCLA Center for the Study of Urban Poverty, Day Laborers in Tokyo, Japan : Preliminar Findings from the San'ya Day Labor Survey, Working Paper Series, 2000.

12 - Jeux de hasard entre le flipper et la machine à sous. 
identité. Paradoxalement, ils s'attribuent tous la responsabilité de leur situation, même s'ils ont conscience d'avoir fait les frais de la crise économique. L'histoire damnée de Sanya, qui condamne ce quartier à une réputation de ghetto où sont concentrés des pauvres, des asociaux et des marginaux, renvoie à ses habitants une image dévalorisée d'eux-mêmes. Les sans-abri de Sanya semblent intérioriser, au moins partiellement, le jugement accusateur et le mépris que les Japonais «ordinaires» portent sur eux.

Toutefois, malgré le poids de la discrimination sociale et du vécu de la misère, on aurait tort de s'attarder exclusivement sur les aspects négatifs de leur condition et de l'image qu'ils ont d'eux-mêmes. Car les efforts qu'ils font pour préserver leur dignité sont proportionnels à leur peur de la perdre. Dans tous les entretiens, l'exigence de dignité est au cœur de la problématique identitaire de ces hommes. Plus que la survie, leur souci majeur est de ne pas franchir les limites au-delà desquelles ils pourraient cesser d'être à leurs propres yeux un être humain digne de ce nom.

\section{Le «nouveau» problème des sans-abri ${ }^{13}$}

L'exigence de dignité n'est pas le seul fait des sans-abri de Sanya, elle est partagée par la grande majorité des sans-abri de Tokyo. Le fait que tous reproduisent le mode d'organisation typique des habitations japonaises, comme de retirer ses chaussures à l'entrée de leur cabane ou de leur tente par exemple, en est un reflet.

J'ai passé beaucoup de temps avec les sans-abri du centre de Tokyo, notamment par le biais de l'association NOJIREN créée et gérée par les sans-abri eux-mêmes. Ses membres forment un groupe dont le noyau regroupe une quinzaine de personnes très soudées. Ils se sont réunis autour d'une cause, la leur, et ont en commun la volonté de combattre politiquement pour leur dignité, et de lutter contre l'isolement des autres sans-abri. Ils mènent les activités de l'association tous les jours, la plupart travaillant la nuit en récupérant des cannettes vides qu'ils revendent au poids. Certains se sont installés dans le parc Yoyogi, immense espace vert au cour de Tokyo: leurs tentes de bâches bleues sont regroupées et organisées avec un "coin» cuisine, une tente commune pour déposer les objets récupérés et une autre pour les cannettes. D'autres sont installés à Shibuya, dans un petit parc où ils ont construit de véritables cabanes en bois. Trois fois par semaine, ils organisent des «patrouilles» dans les parcs, au cours desquelles ils vont voir tous les sans-abri ou nakama ${ }^{14}$ qu'ils croisent, pour discuter, leur apporter de la chaleur, et les informer des événements ${ }^{15}$ organisés par l'association ou des mesures les concernant. Tous les samedis soir, ils organisent un takidashi, un repas chaud en plein air, qu'ils distribuent à une centaine de personnes, voire le double. Dès la fin du repas s'organise la "patrouille» de nuit. Munis de médicaments (offerts par Médecins sans frontières), d'une trousse d'urgence, de couvertures de survie, et d'un thermos avec

13 - Le terme sans-abri me semble le plus neutre pour désigner cette population, bien que le terme japonais nojuku seikatsusha [personne qui vit dehors] soit plus approprié.

14 - Nakama [collègue, camarade, copain] : terme que les sans-abri emploient pour se désigner les uns les autres.

15 - Manifestations pour réclamer du travail, protester quand l'un d'entre eux a été tué, etc. 
du thé chaud, plusieurs groupes de trois ou quatre personnes vont sillonner les rues pour s'assurer que tous les nakama vont bien. Une fois par mois un médecin volontaire vient pour offrir gratuitement ses services aux sans-abri qui le souhaitent.

Les derniers chiffres officiels ${ }^{16}$ recensent 25300 sans-abri au Japon dont 6360 à Tokyo et 7760 à Osaka. Leur situation comporte de nombreuses spécificités qui contrastent avec celles des SDF en France. La plus remarquable est le nombre important de personnes âgées : $80 \%$ d'entre eux ont plus de 50 ans. Contrairement aux SDF français, dont un tiers aurait entre 18 et 29 ans, moins de $1 \%$ des sans-abri japonais appartient à cette tranche d'âge. C'est une population majoritairement masculine, les femmes représentant moins de $5 \%$ des sans-abri, et les familles avec enfants sont quasi inexistantes $(0,1 \%)$.

L'organisation du lieu de vie offre un contraste tout aussi remarquable avec ce que l'on voit en France. Bien que l'organisation des membres de NOJIREN ne soit pas représentative de la population des sans-abri, la plupart vivent tout de même de façon groupée dans des parcs ou sur les bords de rivière, ce qui donne à ces lieux des airs de petits villages. Ainsi, une grande majorité ( $85 \%$ ) vit dans un lieu fixe, dont près de $50 \%$ dans les parcs publics et $18 \%$ sur les bords de rivière. Plus de la moitié d'entre eux s'est construit un lieu en «dur»: une maisonnette, faite le plus souvent en bois, ou une tente améliorée recouverte de bâche bleue (blue sheets). Ces «villages bleus» dans les parcs et sur les berges inscrivent chacun, par volonté ou nécessité, dans le réseau de connaissance et de solidarité du lieu. Cette particularité ne doit pas nous faire oublier ceux qui vivent de façon isolée et/ou qui se contentent d'une couverture ou d'un abri improvisé en carton chaque nuit.

Près de $65 \%$ des sans-abri travaillent, la majorité (environ $75 \%$ ) dans la collecte de cannettes ou journaux pour le recyclage, ou en tant que travailleurs journaliers $(20 \%)$.

Bien qu'il y ait un terme pour dire "personne sans domicile fixe», il est très rarement employé, sans doute parce que la grande majorité vit justement dans un lieu fixe (voir note 13). Le terme homeless est le plus couramment employé, même s'il ne définit pas mieux les sans-abri que les termes japonais nojuku seikatsusha [personne qui dort dehors] ou rojô seikatsusha [personne qui vit dans la rue]. C'est pourtant cette désignation anglophone qui devient le terme officiel depuis la parution du livre blanc sur les sans-abri de Tokyo en $2001{ }^{17}$. L'emprunt d'un terme étranger pour désigner les sans-abri renforce la notion de nouveauté et d'étrangeté, comme s'il s'agissait d'un phénomène venu de l'extérieur qui aurait soudainement «contaminé» le Japon.

La question des sans-abri attire l'attention depuis 1993-1994. Vers 1997-1998, on commence à parler du «nouveau problème des homeless » : en réalité ce "nouveau problème» n'est pas la conséquence de l'apparition des sans-abri au Japon, mais

16 - Hômuresu no jittai ni kansuru zenkoku chôsa [Enquête nationale sur les conditions des sansabri], Ministère des Affaires sociales, 2003 : www.mhlw.go.jp/houdou/2003/03/h0326-5.html.

17 - Tôkyô no hômuresu [Les sans-abri de Tokyo], Tôkyôto fukushi kyoku [service d'aide social de la mairie de Tokyo], 2001. 
celle de leur récente visibilité urbaine. Il y a toujours eu des sans-abri au Japon, ils vivaient dans les quartiers pauvres et particulièrement dans les yoseba, mais leur brutale augmentation a fait déborder le phénomène au cour des villes, et a ainsi conduit la population à prendre conscience de leur existence, jusque-là ignorée par la grande majorité. $\mathrm{Si}$, comme le rappelle le livre blanc, les "yoseba avaient pour fonction d'absorber les hommes qui avaient perdu leur travail stable ${ }^{18}$ ", cela n'a jamais empêché ces derniers de se retrouver à la rue, contrairement à ce que souligne ce même rapport.

D'après le livre blanc, $60 \%$ des sans-abri étaient des travailleurs journaliers avant de se retrouver à la rue : ils sont considérés comme les «anciens" sans-abri, contrairement aux "nouveaux» qui avaient un travail régulier comme en témoigne le phénomène des "sans-abri en costume» (suit homeless) - d'anciens cadres ou employés qui se retrouvent à la rue du jour au lendemain, généralement à la suite d'un licenciement ou d'un problème familial, et qui, par honte ou par refus de leur nouvelle condition, cherchent à s'en cacher aux yeux de la société en gardant leur costume de travail. Certains continuent à travailler, mais ils ne peuvent louer un logement faute de ressources suffisantes. Il n'est pas rare de voir un homme en costume rentrant du travail se coucher dans une boîte en carton pour y dormir.

\section{Visibilité et invisibilité de la pauvreté}

Ce qui pose problème n'est pas l'existence de la pauvreté, mais sa visibilité. Elle dérange profondément. Les multiples expulsions forcées des sans-abri des lieux publics pour divers prétextes (travaux, plaintes de la population...) constituent sur le plan symbolique "une "dépollution" de l'espace urbain ${ }^{19}$ ». Les expulsions "temporaires" dès qu'une personnalité étrangère se rend au Japon montrent bien les efforts $\mathrm{d}^{\prime}$ " invisibilisation» mis en œuvre par le gouvernement; des travaux sur l'invisibilité sociale montrent que les processus de constitution du visible opèrent une hiérarchie dans ce qui peut être vu ${ }^{20}$. Entre 1994 et 2000, il y aurait eu vingtneuf expulsions forcées de sans-abri au Japon ${ }^{21}$. Deux d'entre elles, en 1994 et en 1996, ont profondément secoué le pays. Celle de 1996 concernait plus de 200 sansabri installés dans le hall qui mène de la gare de Shinjuku à la mairie de Tokyo et au quartier des affaires; la mairie imposa l'expulsion pour la construction d'un passage roulant. Le 24 janvier, face à la résistance des sans-abri soutenus par des associations, 400 gardes et policiers effectuèrent l'expulsion. Beaucoup de Japonais se sont dits choqués par la violence de l'opération. Cette année-là, la presse publia un nombre record d'articles sur les sans-abri.

Les sans-abri, figure la plus extrême de la pauvreté, ne sont que la partie visible de l'iceberg: les allocataires de l'assistance publique (l'équivalent de nos «RMIstes»),

\footnotetext{
$18-$ Ibid.

19 - S. Roy et D. Laberge, "Pour être il faut être quelque part», Sociologie et Sociétés, vol. 33, n 2, 2001.

20 - O. Voirol, «Visibilité et invisibilité : une introduction», Réseaux, n 129-130, Paris, Lavoisier, 2005.

21 - Rapport des ONG sur l'engagement international des droits économiques, sociaux et culturels, 2000, http://member.nifty.ne.jp/nojuku/english/NGOrep.html.
} 
beaucoup plus nombreux, ne sont pas «repérables» dans l'espace public et ne bénéficient pas de la visibilité médiatique des freeters.

Les statistiques de l'assistance publique rendent compte d'une "pauvreté qu'on ne voit pas ${ }^{22}$ » et les différentes catégories d'allocataires donnent une idée des autres populations touchées par la pauvreté : les personnes âgées sont la catégorie la plus représentée ( $46,4 \%$ des allocataires), suivie des "personnes handicapées ou malades» $(35,8 \%)$, et enfin des «mères seules» (familles monoparentales) $(8,4 \%)$.

Cette présentation des différentes formes de pauvreté urbaine est loin d'être exhaustive. Beaucoup d'individus sont aussi concernés parmi les descendants des burakumin [parias] ${ }^{23}$, les populations immigrées ou celles touchées par le chômage dont le retour à l'emploi est incertain.

\section{Les représentations de la pauvreté}

Pour Serge Paugam, la sociologie de la pauvreté est née avec les travaux de Tocqueville et de Simmel ${ }^{24}$. Tocqueville soulignait le caractère relatif de la pauvreté : être pauvre dans un pays pauvre n'a pas le même sens qu'être pauvre dans un pays plus prospère. Pour Simmel, la pauvreté est «aussi construite socialement. Son sens est celui que la société lui donne ${ }^{25}$ ». La pauvreté n'existe pas en soi mais en fonction du regard que la collectivité porte sur elle, regard qui identifie «le pauvre»par rapport à la norme. Selon les cultures et les époques, ce regard varie et modifie le statut du pauvre. Les représentations sociales permettent de rendre compte de la façon dont les membres d'une société "pensent» la pauvreté.

Une représentation n'est pas le pur reflet de la réalité, mais une construction qui renvoie à l'imaginaire social et individuel : elle construit une réalité sociale. Émile Durkheim, qui avait relevé le caractère stable des représentations, soulignait que «seuls les événements d'une suffisante gravité peuvent réussir à affecter l'assiette mentale de la société ${ }^{26}$ ». Les "événements d'une suffisante gravité» seraient capables de produire un changement au sein d'une représentation établie. Il peut s'agir d'un changement de conjoncture économique (apparition du chômage), d'un nouveau phénomène social (visibilité des SDF) ou encore d'une image véhiculée par les médias. Leur impact est d'autant plus important qu'ils sont relayés par l'éducation.

22 - O. Aoki (dir), Gendai nihon no «mienai» hinkon [La pauvreté invisible dans le Japon actuel], Tokyo, Akashi shoten, 2005.

23 - Les burakumin sont victimes d'une discrimination très ancienne qui perdure malgré des lois pour leur émancipation. Ils descendent des eta ["êtres souillés»], spécialisés dans le travail du cuir, et des hinin ["non-humains»] composés de mendiants, de saltimbanques et de gens exclus de leur communauté.

24 - S. Paugam, Les formes élémentaires de la pauvreté, Paris, PUF, 2005.

25 - S. Paugam, «Naissance d'une sociologie de la pauvreté», in G. Simmel, Les pauvres, Paris, PUF, 1998.

26 - É. Durkheim (1912), Les formes élémentaires de la vie religieuse. Le système totémique en Australie, Paris, PUF, 1968, p. 609. 
C'est ce type d'événements que j'ai tenté de repérer dans la société japonaise à travers les politiques d'assistance publique et la presse, afin de dégager les évolutions de la figure du pauvre.

\section{Les lois d'assistance aux pauvres}

La loi actuelle d'assistance publique, dernier filet de sécurité sociale, a été promulguée en 1946. Elle opère une véritable coupure avec les anciennes lois d'assistance (la première date de 1874) qui concernaient seulement les pauvres invalides - ceux qui n'ont pas la capacité de travailler du fait de leur âge ou d'un handicap - ou ne pouvant "réellement compter sur personne», excluant en conséquence toute personne ayant une famille ${ }^{27}$. De fait, elles établissaient une distinction entre le "vrai pauvre», invalide et sans famille, et le "faux pauvre», valide et responsable de sa condition, qui ne mérite pas l'assistance.

Cette distinction a prévalu dans les lois d'assistance pendant près d'un siècle, et elle a véhiculé une certaine représentation de la pauvreté dont découlait une notion de mérite à l'assistance.

La loi d'assistance publique fût promulguée la même année que la Constitution japonaise, dans un contexte d'après-guerre confrontant le pays à une pauvreté massive contre laquelle les anciennes lois étaient de fait inadéquates. Sous la pression des experts américains, les aspects discriminatoires, arbitraires et préférentiels disparurent de la nouvelle loi, ce qui fut le plus difficile à accepter et à assimiler pour les bureaucrates, mais aussi pour la population. Ainsi la loi d'assistance publique vise, selon l'article 1 «toutes les personnes vivant dans l'indigence» et la distinction entre pauvre valide et pauvre invalide est supprimée. Le jugement de valeur lié à cette distinction ne va pas disparaître pour autant, les avancées juridiques étant plus rapides que le changement des mentalités.

Des statistiques récentes montrent que la part de l'assistance publique dans les dépenses de la sécurité sociale est en baisse continue. Elle était de 7,8 \% en 1970, de 2,7\% en 1990 et de 2,5\% en 2000. Le gouvernement justifie cette baisse par l'expansion économique du pays. Selon cette logique, la part de l'assistance publique aurait dû augmenter durant la crise des années 1990, ce qui ne fut pas le cas. La croissance économique des années 1970 a eu pour effet l'élévation générale du niveau de vie et l'apparition d'une classe moyenne élargie. Se répand alors l'idée d'une «nouvelle masse moyenne», dont les contours flous tendent à inclure tout le monde ${ }^{28}$. La relative homogénéisation du mode de vie a eu pour conséquence l'universalisation de l'État providence, c'est-à-dire que les premiers bénéficiaires sont devenus la classe moyenne, aux dépens des couches défavorisées devenues minoritaires. Ce glissement de l'action de l'État est rendu possible par un déplacement des questions sociales :

27 - La solidarité familiale devient légalement obligatoire dans le Code civil de 1898, imposant aux familles le "devoir d'entretenir» les siens.

28 - B. Bernier, «Le japon, société sans classes?», Anthropologie et Sociétés, vol. 18, n 1, 1994. 
dès 1970, l'Agence de planification économique décrit le Japon comme étant sur le point de dissoudre la pauvreté ${ }^{29}$ et la prospérité économique des années 1980 renforce cette idée. Ce n'est plus l'urgence de la situation précaire mais la notion de «risque» qui est mise en avant. La nucléarisation des familles et le vieillissement de la population occupent alors le devant de la scène des préoccupations sociales. En 2000, 68 \% des dépenses de sécurité sociale concernent les personnes âgées.

\section{L'assistance publique aujourd'hui}

Les personnes âgées ou handicapées représentent 84,2 \% des foyers d'allocataires de l'assistance publique. Sans doute trouvent-elles dans leur infirmité ou leur âge une justification "légitime» à leur recours à l'assistance et en font-elles plus facilement la démarche. Vient ensuite la catégorie des "mères seules», et enfin la catégorie "autres", qui représente $7 \%$ des allocataires. Les «autres» sont en réalité ceux qui ne sont "que pauvres», comparés aux autres catégories désignées par le «désavantage» qui s'ajoute à leur pauvreté; ils sont à la limite de l'inexistence en raison du flou qui les désigne et de leur proportion très minoritaire. Pourtant, ce sont justement les "personnes dans l'indigence» qui sont la cible de l'assistance publique. Deux facteurs peuvent expliquer ce faible taux d'allocataires "autres» : soit ces derniers, par peur du stigmate ou par méconnaissance de leurs droits, ne demandent pas l'assistance publique, soit les pratiques administratives, se référant à la traditionnelle distinction entre pauvre valide non méritant et pauvre invalide, les écartent de leur droit. La faible proportion de jeunes allocataires (17\% seulement ont entre 20 et 49 ans) est la conséquence directe de ces pratiques qui tendent à refuser l'aide aux personnes capables de travailler.

Ce qui pose problème dans l'attribution de l'assistance est dû en partie à l'ambiguïté de certains articles de la loi. C'est le cas notamment de l'article 4 sur le "caractère strictement supplémentaire de l'assistance», selon lequel "toutes les ressources et capacités de l'individu doivent être utilisées» avant de pouvoir prétendre à cette aide.

Dans les guides pour les fonctionnaires de l'aide sociale, cet article est interprété par l'obligation de vérifier systématiquement les «ressources» (entendues comme les revenus, les avoirs et les biens) et la "capacité de travailler». Pour juger de cette capacité, les guides s'appuient sur deux éléments : l'âge, et la validité ou l'invalidité du demandeur. Si selon ces critères, le demandeur est considéré capable de travailler, les guides préconisent alors de juger de sa volonté de travailler. Un procédé pour le moins choquant : comment cette appréciation peut-elle ne pas être subjective et arbitraire? Cette étape est cependant décisive, car d'elle dépend l'acceptation des demandeurs (valides) : si le fonctionnaire estime que le demandeur ne veut pas travailler, ce dernier n'aura pas accès à l'assistance publique. Pour juger de cette volonté, les guides proposent de s'appuyer sur la fréquentation de hello work (équivalent de l'ANPE). Mais reste aux fonctionnaires de décider du nombre raisonnable de visites à cet organisme pour se prononcer sur la motivation de l'individu.

29 - Keizai Hakusho [Livre blanc sur l'économie], cité par M. Chûbachi et T. Kôji, in Hugh Patrick (dir.), Japanese Industrialization and Its Social Consequences, Berkeley, University of California Press, 1976, p. 431 
Cette interprétation est un véritable contournement de la loi, et du principe fondamental de l' «égalité de toutes les personnes démunies», stipulé dans l'article 1. La loi semble malléable : entre les interprétations, les contournements ou la mobilisation de certains articles pour justifier les diverses pratiques, elle perd de son autorité.

Le fait que seule une petite proportion de pauvres bénéficie de cette aide témoigne de l'ampleur de ces pratiques: le taux d'assistance, qui mesure la proportion d'allocataires par rapport à la population totale, est descendu de 1,18 \% en 1985 à $0,7 \%$ en 1995 et n'est repassé au dessus de la barre des $1 \%$ de la population qu'en 2003 (1,05\%). Le taux de pauvreté qu'affiche le Japon dans les récentes statistiques du PNUD ou de l'OCDE (soit 11,7 \% et 15,3\%) offre un écart conséquent avec ce taux d'assistance. Officiellement, il n'existe pas de taux mesurant la proportion d'allocataires par rapport aux personnes éligibles à l'assistance publique, mais plusieurs chercheurs l'ont calculé, conscients du fait que beaucoup de foyers éligibles ne percevaient pas cette aide. Selon le dernier taux connu (1999), les allocataires ne représenteraient que 19,6 \% de la population éligible.

Plusieurs cas de morts violentes (de faim ou de froid) de personnes à qui l'on avait refusé l'assistance publique ou de recours en justice contre l'administration face à des «refus abusifs» ont fait l'objet d'articles dans les quotidiens. Lors de mon enquête de terrain auprès des sans-abri, j'avais déjà eu plusieurs échos concernant ces pratiques administratives illégales, qui pouvaient aller jusqu'à refuser l'assistance à des sans-abris sous prétexte qu'ils n'avaient pas atteint 65 ans ou qu'ils n'avaient pas de domicile.

En 2001, face à l'augmentation continue de ces refus, le ministère de la Santé et des Affaires sociales a fait circuler une notification à ce sujet dans les bureaux d'assistance publique: "Même les sans domicile fixe ou les personnes de moins de 65 ans peuvent recevoir l'assistance publique ${ }^{30}$.» Cette circulaire témoigne indirectement de la reconnaissance de pratiques inadmissibles dans l'attribution de l'assistance, et de la volonté de les changer.

\section{La pauvreté dans la presse écrite}

Afin de repérer le traitement journalistique de la pauvreté, dont découle une certaine figure du pauvre, j'ai constitué une base de données à partir des articles du quotidien Asahi Shimbun (deuxième quotidien national) sur le thème de la pauvreté entre 1990 et 2002. Le principal constat de cette étude est que les articles dont le titre contient le terme pauvreté (hinkon) n'apparaissent que dans la rubrique internationale. Il n'est donc employé que pour parler de l'étranger.

Comment penser l'absence du terme "pauvreté» dans les articles sur le Japon? Cette question est intrinsèquement liée à la perception de la pauvreté "japonaise», car lorsqu'il s'agit des autres pays, la pauvreté est facilement reconnue et l'usage du terme ne pose aucun problème. Dans les articles sur le Japon, ce sont des termes décrivant 
des catégories de pauvres qui sont employés, tels que «sans-abri»" «travailleur journalier», etc. Ainsi, il existe bien des sans-abri, des allocataires de l'assistance publique, des travailleurs journaliers et d'autres catégories de personnes qui «vivent difficilement», mais il n'y a pas de pauvres. Ces différentes situations ne sont pas comprises comme un même phénomène. Le non-usage du terme "pauvreté», tout comme le fait qu'un taux de pauvreté national fasse défaut, entretient le mythe de l'absence de celle-ci.

\section{La définition de la pauvreté}

Les représentations véhiculées par le système d'assistance publique et par le quotidien dont nous avons fait l'étude ont sans aucun doute exercé une influence sur celles de la population, et sur ce que cette dernière entend par le terme "pauvreté» ou sur les populations qu'elle considère comme pauvre.

Une équipe de chercheurs a effectué une enquête inédite sur les perceptions de la pauvreté, auprès d'étudiants en aide sociale ${ }^{31}$ (ci-après "étudiants") et d'habitants d'un quartier d'une ville d'Hokkaido (ci-après "habitants") ${ }^{32}$.

L'un des principaux constats de cette étude est que les sans-abri et les allocataires de l'assistance publique ne sont pas nécessairement considérés comme pauvres: «La vie des sans-abri» n'est associée à la pauvreté que pour 70,8\% des étudiants et 34,8\% des habitants. De même, seuls 52,5\% des étudiants et 27,8\% des habitants associent «la vie des allocataires de l'assistance publique» au terme "pauvreté». Les situations les plus souvent associées à la pauvreté sont «la vie au Japon avant la guerre et juste après la guerre» et «la vie dans les pays en voie de développement ou en guerre» ${ }^{33}$.

Le terme "pauvreté» (hinkon) a été volontairement utilisé dans cette enquête. En japonais, il existe plusieurs adjectifs et expressions (seikatsu ni komaru : "avoir des difficultés pour vivre», girigiri no seikatsu : «avoir du mal à joindre les deux bouts») pour exprimer des difficultés financières plus ou moins graves; l'usage du terme "pauvreté» est limité au domaine de l'histoire et aux pays pauvres.

L'enquête se termine par la question "Avez-vous ressenti une gêne ${ }^{34}$ en entendant le mot "pauvreté" ?» : plus de $45 \%$ des étudiants et près de $53 \%$ des habitants ont répondu par l'affirmative. Il leur était alors demandé de dire pourquoi, et la grande majorité des réponses citent l'ambiguïté de la définition du terme "pauvreté» : "Je ne comprends pas le terme pauvreté.» «Il y a la pauvreté affective, intellectuelle ou monétaire, de quelle pauvreté est-il question ici?» «De quelle pauvreté voulez-vous parler?» «Il y a une différence entre pauvre (mazushii) et pauvreté (hinkon).» "Je n'arrive pas

31 - Les universités japonaises proposent aujourd'hui des départements d'«aide sociale» (shakai fukushi).

32 - O. Aoki, "Gendai nihon no "hinkonkan" ni kansuru anketo kekka chûkan hôkoku», Kyoiku fukushi kenkyu, $\mathrm{n}^{\circ} 12,2006$.

33 - Entre 75 et $88 \%$ des étudiants et des habitants citent ces réponses.

34 - Iwakan: manque d'harmonie, malaise, désaccord. 
à me représenter la pauvreté.» "C'est un terme abstrait.» "Parce qu'il n'y a pas de pauvreté dans le Japon actuel.» «Je ne peux associer le terme "pauvreté" au Japon.»

Les résultats de cette enquête ne signifient pas que les personnes interrogées ne savent pas dissocier les situations de pauvreté d'autres situations, mais que le terme japonais hinkon (pauvreté), n'étant plus utilisé pour décrire ces situations dans le Japon actuel, ne signifie plus ce qu'il signifie intrinsèquement. Il est associé à des images lointaines (historiques ou étrangères) qui ne font pas référence à l'expérience individuelle.

Dans la vie courante, ce sont des formules moins «crues» qui sont employées, et celles-ci peuvent aussi bien se rapporter à des situations extrêmes (comme celle des sans-abri) qu'à des situations plus "anodines» (décrire le fait de ne pas avoir d'épargne ou de ne pas avoir d'argent sur soi). Ainsi l'ambiguïté de ces expressions courantes ne permet pas de dissocier l'acceptable de l'inacceptable, ni de rendre compte de la pauvreté au sens propre du terme.

\section{Le droit à l'assistance}

L'existence de pratiques arbitraires dans l'attribution de l'assistance ne peut expliquer à elle seule le très faible taux d'allocataires: des personnes éligibles n'en font aussi pas la demande, ou ne tentent pas de recours après un refus abusif. Dans les deux cas, ces situations témoignent d'une méconnaissance ou d'une faible conscience du droit de l'individu à l'assistance. Comment expliquer que celle-ci ne se soit pas élevée avec la modernisation, la démocratisation du pays et la constitutionnalisation du droit? Que le pouvoir ne mène aucune action pour informer les populations démunies de leurs droits est un frein incontestable au développement de l'assistance. Même dans les bureaux d'assistance publique et en particulier dans les salles d'attente, il n'y a aucune information à disposition des usagers : ni brochure ni dépliant ni affiche sur le système d'assistance publique, ni sur quoi que ce soit d'autre d'ailleurs. Pourtant, les autres services d'aide sociale ont leur lot de tracts et d'informations diverses concernant le service en question. D'une façon générale, pour obtenir des informations, il faut aller se renseigner directement dans les bureaux d'assistance publique aux guichets desquels on vous remet un numéro pour passer un "entretien" avec un conseiller plutôt que de vous donner le fascicule ou même un formulaire de demande ${ }^{35}$.

Le chapitre 8 de la loi d'assistance publique, qui a pour titre «Droits et devoirs des assurés", ne comporte en réalité que des articles sur les devoirs des allocataires et sur ce qui leur est interdit. Aucune mention du droit à l'assistance ne figure dans cette loi, bien que celui-ci soit stipulé dans la Constitution. On peut y voir, si ce n'est une forme de déni, tout du moins une position ambiguë par rapport à la reconnaissance du droit des pauvres à l'assistance.

35 - Ce qui explique la multiplication de la publication d'ouvrages de type "manuel d'assistance publique» depuis 2000-2001, qui se vendent très bien. 
Dans les entretiens que j'ai menés avec les fonctionnaires de l'assistance publique, les discours concernant l'accès à l'assistance révèlent que la notion de "droits » a des contours flous. L'affirmation du droit à l'assistance est toujours suivie d'une nuance qui la remet en cause : "C'est un droit, mais avant le droit, il y a quand même la responsabilité individuelle, non?»

Le statut du droit, en tout cas du droit social, n'est pas intégré dans les mentalités. Le droit est une règle extérieure, une façade. Je voudrais citer ici Toshiaki Kozakai, chercheur en psychologie sociale: «Nous avons tendance à nous conformer à la majorité et à nous opposer à la minorité. Mais étant admis sans discussion, le point de vue dominant est peu intériorisé et paradoxalement faible car il ne suscite aucun travail cognitif. L'influence majoritaire est superficielle car elle ne repose que sur une mise en conformité ${ }^{36}$.»

Cette remarque est tout à fait significative pour l'analyse de la conception du droit social. Il faut rappeler le contexte de l'élaboration de la loi d'assistance publique et la pression faite par les experts américains pour débarrasser la loi des critères moraux et discriminatoires. Cette évolution majeure offre un nouveau visage au cadre de l'assistance et aux «assistés». Mais un tel changement des conceptions de l'aide et de la pauvreté implique un travail d'éducation et de sensibilisation à ces nouveaux principes, pour que ces nouvelles valeurs puissent être intégrées par la population. Or il n'y a pas eu de tel travail pour prendre le relais du cadre légal ${ }^{37}$. C'est la loi, et on ne remet pas en question la loi. Mais sans questionnement ou «travail cognitif», les notions légales ne peuvent être assimilées; les valeurs sousjacentes au droit ne sont pas acquises.

Toutefois, l'année 2001 marque un tournant significatif dans les mesures d'assistance et le droit des pauvres. Le ministère des Affaires sociales a entrepris de changer les pratiques discriminatoires des fonctionnaires en faisant circuler une notification rappelant la loi. Surtout, une nouvelle loi est établie pour les sans-abri. Promulguée le 7 août 2002, la "loi de Réinsertion sociale des Homeless" marque une rupture avec l'attitude des institutions envers les sans-abri. Son premier article énonce plusieurs éléments fondamentaux: la reconnaissance de la non-responsabilité individuelle des personnes devenues sans-abri («du fait de circonstances inévitables») ainsi que celle de leurs droits; l'affirmation du devoir de l'État de soutenir l'assistance aux sans-abri et enfin, la nécessité de "compréhension» de la population face à cette question. Ces principes témoignent d'un retournement de la conception du phénomène des sans-abri et de la volonté d'en changer la perception négative dans la population. Les mesures sont destinées à améliorer non seulement les conditions de vie des sans-abri, mais aussi celles des individus «à risque». Il y a une prise en compte du phénomène plus global de la pauvreté au sein de cette loi. L'existence d'une misère extrême est reconnue, sans que celle-ci soit nommée : il est

36 - T. Kozakai, entretien dans la revue Synapse, $\mathrm{n}^{\circ} 170$, novembre 2000.

37 - Malgré quelques travaux très intéressants (voir en bibliographie Aoki, Bitô, Iwata, Malinas...), dont l'impact est cependant souvent limité au milieu universitaire. 
par exemple question des "personnes qui risquent de devenir sans-abri», mais pas de "pauvres» ou de "précaires».

L'article 2 sur la "définition" des sans-abri pose cependant problème : "Le terme homeless dans cette loi désigne les personnes qui vivent illégalement et quotidiennement dans les parcs, les berges, les gares, sur les routes ou autres équipements.» Le sans-abri est défini comme un "parasite». Se situant dans l'illégalité, il apparaît également comme un délinquant: sa seule et inévitable présence constitue en elle-même un délit. Il y a donc d'une part l'absence des qualificatifs fondamentaux du homeless (tel "qui n'a pas de logement»), mais surtout une criminalisation du sans-abri. Une telle définition «légale», sur laquelle repose un texte de loi destiné à l'assistance et à la réinsertion sociale des sans-abri, amène à mettre en doute les objectifs premiers de cette loi.

L'article 11 sur "l'utilisation appropriée des lieux publics» autorise l'expulsion «légale» des sans-abri, alors qu'en 1993 le Japon a signé la «Résolution concernant les expulsions» des Nations unies. Soupçonnant que cet article soit le but caché de la loi, les organisations de soutien aux sans-abri s'y sont violemment opposées, dénonçant cette mesure comme contraire aux droits de l'homme.

Fondamentalement, rien ne justifie le fait de créer une loi spécifique pour les sans-abri, puisque la loi d'Assistance publique, si tant est qu'elle soit appliquée à la lettre, se prête aussi bien aux sans-abri; l'ajout de mesures spécifiques dans les clauses additionnelles pourrait suffire. Malgré ces ambiguïtés, cette loi apparaît toutefois comme une avancée sensible dans la reconnaissance de l'existence de pauvreté, que l'on se doit de prendre en compte comme telle dans l'évolution des politiques d'assistance envers les pauvres.

\section{Conclusion}

L'analyse de l'assistance publique montre le fossé entre la loi et sa mise en application. Les anciennes lois ont entériné des conceptions de la pauvreté qui perdurent dans les pratiques d'attribution de l'assistance: celles-ci reposent sur la notion de mérite et distinguent le "vrai pauvre» du pauvre valide. L'absence d'un taux de pauvreté national, le très faible taux d'allocataires et le non-usage du terme «pauvreté» entretiennent le mythe de l'absence de celle-ci et révèlent un déni ou une «invisibilisation» de son existence.

Michel Messu disait à propos de la redécouverte de la pauvreté en France dans les années 1965-1970: "[...] il y a une véritable difficulté à conceptualiser l'existence de ceux que l'on vient de désigner comme "pauvres" au sein d'une société française qui se perçoit comme une société $\mathrm{d}^{\prime}$ opulence ${ }^{38}$ ». "Conceptualiser» cette existence appelle une réflexion sur la façon dont est appréhendée la pauvreté : son

38 - M. Messu, «Pauvreté et exclusion en France», in F. Merrien (dir.), Face à la pauvreté, Paris, Les Éditions de l'Atelier, 1994, p. 144. 
existence relève-t-elle d'un problème économique, d'inégalité, ou de responsabilité individuelle? Quel statut ont les pauvres dans notre société? Les représentations de la pauvreté qui découlent de ces questions forment la base des orientations des politiques sociales.

Mais un tel travail de conceptualisation ne peut commencer qu'une fois que les pauvres sont identifiés et désignés comme tels. Si ce travail n'est pas encore entamé au Japon c'est bien parce qu'il n'est pas possible de conceptualiser un phénomène dont on ne reconnaît pas ou dont on ne "voit» pas l'existence. La constitution du visible et du non-visible relève d'une dimension normative et politique ${ }^{39}$. La pauvreté n'est appréhendée qu'à travers de nombreuses catégories, identifiées par les administrations, qui font écran à une perception de la pauvreté comme un phénomène global, à sa visibilité et à sa reconnaissance.

Ce n'est qu'à l'aube du Xxi ${ }^{e}$ siècle que se dessinent les prémices d'une évolution dans les représentations de la pauvreté, marquées par la reconnaissance des sansabri à travers une loi établie à leur encontre. Certes, cette loi comporte des aspects très critiquables, mais elle constitue néanmoins une première étape dans le long processus d'acceptation de l'existence de la pauvreté sous ses diverses manifestations. C'est aussi la première fois qu'il est question, dans un texte de loi, de "droits de l'homme» du pauvre.

Un processus d'évolution est donc en cours, pour l'heure au niveau juridique. Il sera probablement long car il ne suffit pas de décréter le droit, le travail complexe d'intériorisation des valeurs liées à celui-ci est tout aussi fondamental. 


\section{Bibliographie}

- Аокі O. (dir.), Gendai nihon no «mienai» hinkon [La pauvreté invisible dans le Japon actuel], Tokyo, Akashi Shoten, 2005.

- Аокг O., "Gendai nihon no "hinkonkan" ni kansuru anketo kekka chûkan hôkoku » [Résultats provisoires de l'enquête sur les perceptions de la pauvreté dans le Japon contemporain], Kyoiku fukushi kenkyu, $\mathrm{n}^{\circ}$ 12, 2006.

- Bernier B, «Lejapon, sociétésans classes?», Anthropologie et Sociétés, vol. 18, n 1, 1994.

- Bitô H. (dir.), Kore ga seikatsuhogo da [C'est ça l'assistance publique], Kyoto, Takasuga Shuppan, 2005.

- CAstel R., Les métamorphoses de la question sociale, Paris, Gallimard, 1995.

- Gonon A., Le monde des travailleurs journaliers japonais, Tokyo, Maison francojaponaise, 1995.

- Fowler E., San'Ya Blues: Laboring Life in Contemporary Tokyo, Ithaca, Cornell University Press, 1996.

- Honneth A., La lutte pour la reconnaissance, Paris, Cerf (Passages), 2002.

- Hours M., «Les sans-abri de Sanya. Le rapport à la misère des anciens travailleurs journaliers", mémoire de japonais, Université Paris-7, 2002.
- Hours M., «Les représentations de la pauvreté au Japon, à travers les politiques d'assistance et la presse», mémoire de sociologie, EHESS, 2003.

- Iwata S., Hômuresu/gendai shakai/fukushi kokka [sans-abri/société contemporaine/Étatprovidence], Kyoto, Minerva Shobo, 2000.

- Inata S. el Nishizawa A. (dir.), Hinkon to shakaiteki haijyo [Pauvreté et exclusion sociale], Kyoto, Minerva Shobo, 2005.

- L'Hénoret A., Le clou qui dépasse : récit d'un prêtre-ouvrier au Japon, Paris, La Découverte, 1993.

- Malinas D., «Exclus et précaires dans le Japon contemporain. Caractéristiques et analyse du phénomène sans-abri au Japon ", Cités, $\mathrm{n}^{\circ} 27,2006$, p. 41-53.

- Messu M., «Pauvretéet exclusionen France», in F. MERrien (dir.), Face à la pauvreté, Paris, Les Éditions de l'Atelier, 1994, p. 139-169.

- Paugam S., La disqualification sociale, Paris, PUF, 1991.

- Simmel G., Les pauvres, Paris, PUF (Quadrige), 1998.

- Voirol O., «Visibilité et invisibilité : une introduction", Réseaux, $\mathrm{n}^{\circ} 129-130$, Paris, Lavoisier, 2005, p. 9-36. 